\title{
Fuel price changes and their impacts on urban transport - a literature review using bibliometric and content analysis techniques, 1972-2017
}

\begin{abstract}
:
While the period of heightened oil prices in 2005-2014 has attracted considerable academic and policy attention, there is scant cross-disciplinary research considering energy price, transport and land use together. In car dependent societies many socio-economically disadvantaged areas often lack public and active transport accessibility. Emerging research focus has been placed on issues of transport equity including effects of fuel prices. We reviewed 45 years (19722017) of publications about fuel price impacts, transport, and urban context, drawn from Web of Science listed publications. Bibliographic citation analysis reveals eight major research clusters with a set of inter-city comparative studies at their centre. The historical evolution, geographical trends, research approaches and the key themes are revealed by context analysis. The paper highlights the need for further studies looking at energy, transport and land use interaction, and suggests a greater focus on transport equity.
\end{abstract}

Keywords: automobile dependence, energy stress, transport equity, transport and society, fuel price, bibliometric analysis, content analysis

\section{Introduction}

Since oil became the dominant transport fuel, its fluctuating price has had a profound impact on transport and land use, especially in automobile dependent societies (Newman \& Kenworthy, 1989). Oil prices are likely to continue affecting transport and land use costs for years to come even with the emergence of alternative energy sources. Higher fuel prices in the 2000-10s have triggered "fuel protests" (Lyons \& Chatterjee, 2002). Other concerns about oil use arise from the environmental effect of carbon emissions, and the dependence on geo-politically unstable oil supply regions (e.g.: the Middle East) (Colgan, 2014). 
This review examines how scholars have interpreted the impacts of oil price fluctuations on human settlements. Academic interest in oil dependence increased when oil became a major source of energy. From 2005 to 2014, oil prices rose to historical high levels. This long price surge reignited research interest on the impact of higher oil prices on mobility and other effects, such as on household income and wellbeing. More recent falls in oil prices might be due to oil exporters' strategic behaviour to pre-empt or postpone carbonconstraining climate change policies (Van de Graaf \& Verbruggen, 2015). Cheaper oil might prolong the economic feasibility of internal combustion technologies (Khan, 2017). Yet for many developed countries, car-based mobility is an established practice structurally entrenched in energy intensive car-dependent urban forms (Mattioli, Anable, \& Vrotsou, 2016).

\subsection{The energy, transport and land use nexus}

The complex interrelationship between energy, transport and land use forms the nucleus of our understandings about sustainable transport. Scholarly opinions on these interactions are diverse, covering both narrow and broad areas of this interaction. This section briefly reviews how energy factors are being considered in land use or transport.

\subsubsection{Transport's oil dependence problem}

Impacts caused by oil price changes are rooted in structural oil dependence, which is likely to remain unless the phase-out of oil as a motor fuel occurs rapidly. Recent energy modelling by the U.S. Energy Information Administration (2016, p. 5) suggests the share of oil in global transport energy use will remain at $80 \%$ under the reference case model in 2040, only a modest drop from the 2012 level of $87 \%$. There is a wide range of studies that concern themselves with transport's dependence on oil, distributed across multiple fields. Energy studies of fuel prices tend to apply economic models (Hamilton, 2013), in 
which the demand and supply of oil ultimately determines fuel price. Fuel demand is fundamentally dictated by a mix of travel need, distance travelled, and economic conditions. This view gave rise to demand-side transport policies such as stronger fuel economy standards (An, Gordon, He, Kodjak, \& Rutherford, 2007; Schipper, 2011), fuel switching policies (e.g. dieselisation (Bonilla, 2009)), spatially efficient urban planning (Karathodorou, Graham, \& Noland, 2010; Kenworthy, Laube, Newman, \& Barter, 1999), and mobility pricing (Czako, 2015). The supply-side is fundamentally related to oil extraction, which is constrained by the physical and technological limits expressed by the notions of "Energy Return on Energy Invested" (ERoEI), or popularly "peak oil". Geopolitics and energy security can also feature strongly as a supply constraint (Kerschner, Prell, Feng, \& Hubacek, 2013). On the other hand, awareness about the environmental impacts of oil use is increasing (Hickman \& Banister, 2014; Schipper, 2011), and price-based policies to reduce carbon emissions have been advanced, such as carbon taxes, particularly in Europe and Canada (British Columbia). More expensive petroleum fuel might help make alternative propulsion technologies, such as electric motors, batteries, regenerative breaking and hydrogen fuel-cells, more competitive. However, as long as driving remains desirable to many and is associated with a sense of freedom and status (Wells \& Xenias, 2015), technological improvements in fuel economy may not offset the continued growth in travel demand (Schipper, 2011; Sperling \& Gordon, 2009). Meanwhile, emerging and transitional economies with rapid economic and population growth are also likely to contribute to greater oil consumption due to increased automobile ownership and usage (Pojani, 2016). The uncertainties outlined above warrants continued study of the mechanisms and strategies for reducing oil dependence in transport. 


\subsubsection{The intricate relationship between transport and land use}

While non-urban or rural areas may be more affected by higher energy prices, the options for mass transit in rural areas are far more challenging than in cities (Coventry, 2011). But the share of world's population that is urbanised now constitutes a majority, and will continue growing (Fuller \& Romer, 2014). Urban form plays a key role in energy efficient transport arrangements within the city (Figueroa, Lah, Fulton, McKinnon, \& Tiwari, 2014). The linkage of energy use to transport (chiefly determined by distance travelled, especially by private vehicles) is closely related to land use and especially urban density (Newman \& Kenworthy, 1989). However, global cities comparisons rarely consider local level accessibility or cultural differences (Ewing et al., 2018). There is also increasing recognition that fossil fuel phase out and carbon reduction efforts could be better achieved at the level of cities, instead of nation states. Cities can use travel demand management to reduce automobile use or encourage shifts to public transport (Khayesi \& Amekudzi, 2011) to active transport (Rendall, Page, Reitsma, Van Houten, \& Krumdieck, 2011), to shared use of cars or to other modes that do not use oil for propulsion (R. Gilbert \& Perl, 2012).

\subsubsection{Fuel price impact studies in urban areas and transport equity}

Cities are unequal. Mobility and accessibility levels differ across income groups and geographic locations. This leads to the questions of how, and why fuel price impacts are unevenly distributed, or the social equity argument. Equity here refers to "the distribution of impacts (benefits and costs) and whether that distribution is considered fair and appropriate"(Litman, 2018, p. 2). Transport equity concerns the disparities of access to human needs, such as employment, goods, services, activities, and/or social connections (Hine \& Mitchell, 2001; Martens, 2012). Various terms that capture the equity dimension in transport have been developed. Related terms include "transport disadvantage" (Currie 
\& Delbosc, 2011; Denmark, 1998), “social exclusion” (Hine \& Mitchell, 2001; Jeekel, 2014; Social Exclusion Unit (SEU), 2003), and the broader notion "transport poverty" (Lucas, Mattioli, Verlinghieri, \& Guzman, 2016) which combines unaffordable transport costs and disproportional exposure to transport externalities. A sub-set of these terms has focused on the combined impacts of higher fuel costs and car dependence. These terms include "oil vulnerability" (Dodson \& Sipe, 2007), "car dependent economic stress" (Mattioli, Wadud, \& Lucas, 2018), and "fuel poverty in transport" (Berry, Jouffe, Coulombel, \& Guivarch, 2016).

\subsection{Aims and significance of the review}

Despite significant research on the relationship of fuel price, income levels and vehicle travel behaviour (Goodwin, Dargay, \& Hanly, 2004), there is a lack of comprehensive understanding of the interaction of fuel prices, transport and land use. The research areas that are a focus of this review are summarised in Figure 1.

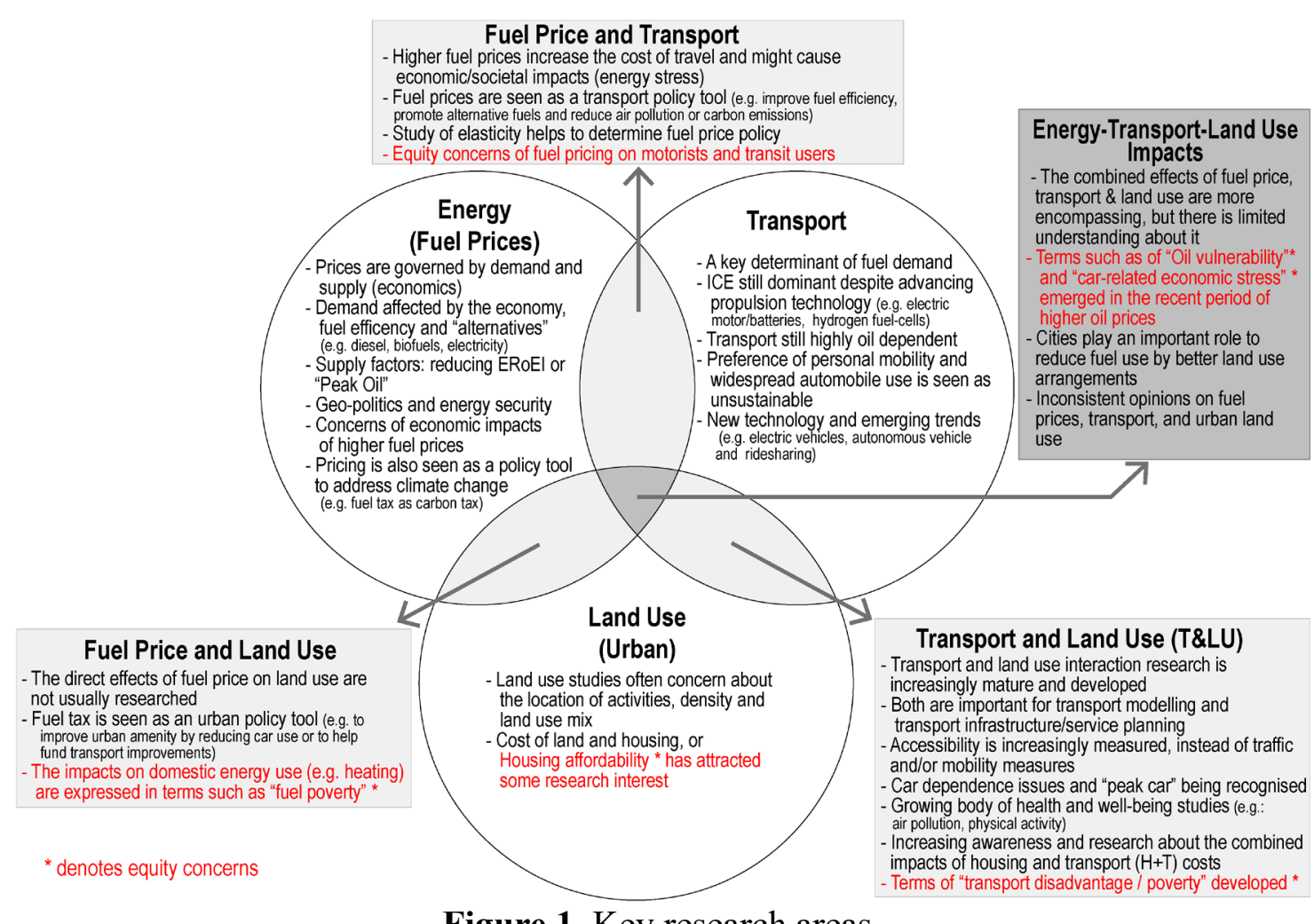

Figure 1. Key research areas. 
We reviewed 45 years of publications investigating fuel price impacts, transport, and urban context, drawn from Web of Science listed publications. Scanning the literature over this longer period helps to understand changes in the research field between the oil "crisis" of the 1970s with its high oil prices, through the long periods of cheap fuel and more recent price spikes. The review sheds light on the historical evolution of such fuel price studies, conceptualising the findings, and may help us to be more resilient in future. Our aims were:

- To map the knowledge development and trajectory of efforts to understand fuel prices, transport and land use, and their relationships

- To reveal key research gaps

- $\quad$ To help synthesise knowledge of the overlapping areas

- To develop future research directions

We outline our methods in Section 2. In Section 3 we present the findings of our analysis. In Section 4 we discuss pathways for future research in this area, focusing on methodological issues, new trends, and possible implications. We conclude the paper with a brief outline of the contributions and limitations made.

\section{Methods}

\subsection{Selection criteria}

The review targeted the fuel price impacts literature from 1970 to 2017, focusing on studies that reference urban areas. The criteria for literature selection is shown in Table 1. Studies that analyse rural areas as well as cities (i.e. urban-rural comparisons) were included. 
Table 1. Criteria for literature selection.

\begin{tabular}{|l|l|}
\hline \multicolumn{1}{|c|}{ Criteria } & \multicolumn{1}{c|}{ Keyword combinations } \\
\hline Petroleum fuel price impacts & $\begin{array}{l}\text { fuel, petrol, gasoline, oil, price, energy, impacts, stress, } \\
\text { affordability, vulnerability }\end{array}$ \\
\hline Transport & transport, travel, commute, journey, mobility \\
\hline Land use (focus on urban areas) & urban, city / cities, town(s), suburb, peri-urban \\
\hline
\end{tabular}

In January 2018, a keyword search was conducted in academic databases, including Transport Research International Documentation (TRID), Google Scholar, Scopus and Thompson Reuters Web of Science (WoS), yielding up to 2,000 matching records. After reading the abstracts and the keywords of these records, 815 were selected and downloaded for further examination. Of these, 470 were found to meet all the selection criteria and investigated in depth. It is this set of papers that helps guide the subsequent discussion section of the paper (from Sections 4 to 5). 320 publications of these publications were contained in the WoS. This sub-set was selected for the further bibliographical citation analysis as part of the review and it is this sub-set that forms the bulk of the results. WoS was used because of its ability to download complete citation records and its better archiving of papers prior to the 1990s. One of WoS's limitations is that it predominately contains journal articles, which are often developed from earlier working/conference papers. Early forms of these papers that were also published were treated as duplications and removed, as were editorial contributions to journal special issues.

\subsection{Bibliographic citation analysis}

We first analyse the overall historical and geographical trends in the literature with descriptive statistics. Then, bibliographic citation analysis is used to show the linkages and researcher interactions. This helps visualise the sub-field and its intellectual interconnections. 
In bibliographic citation analysis, the number of interactions can reflect the level of influence of particular contributions to the literature. The key bibliographic metrics are illustrated in Figure 2. The "direct citation" metric (where Paper A cites Paper B) can show the citation flow between two articles, but it omits linkages where a third article may cite both articles. This can be addressed by the "co-citation" metric (where Paper C cites both Papers A and B). However, a more insightful metric is the "weighted-direct citation" (WDC), which combines both methods by assigning the co-citation value as weights into the direct citation value (Newell \& Cousins, 2015; Persson, 2010). We adopt the WDC metric for citation analysis in this review.
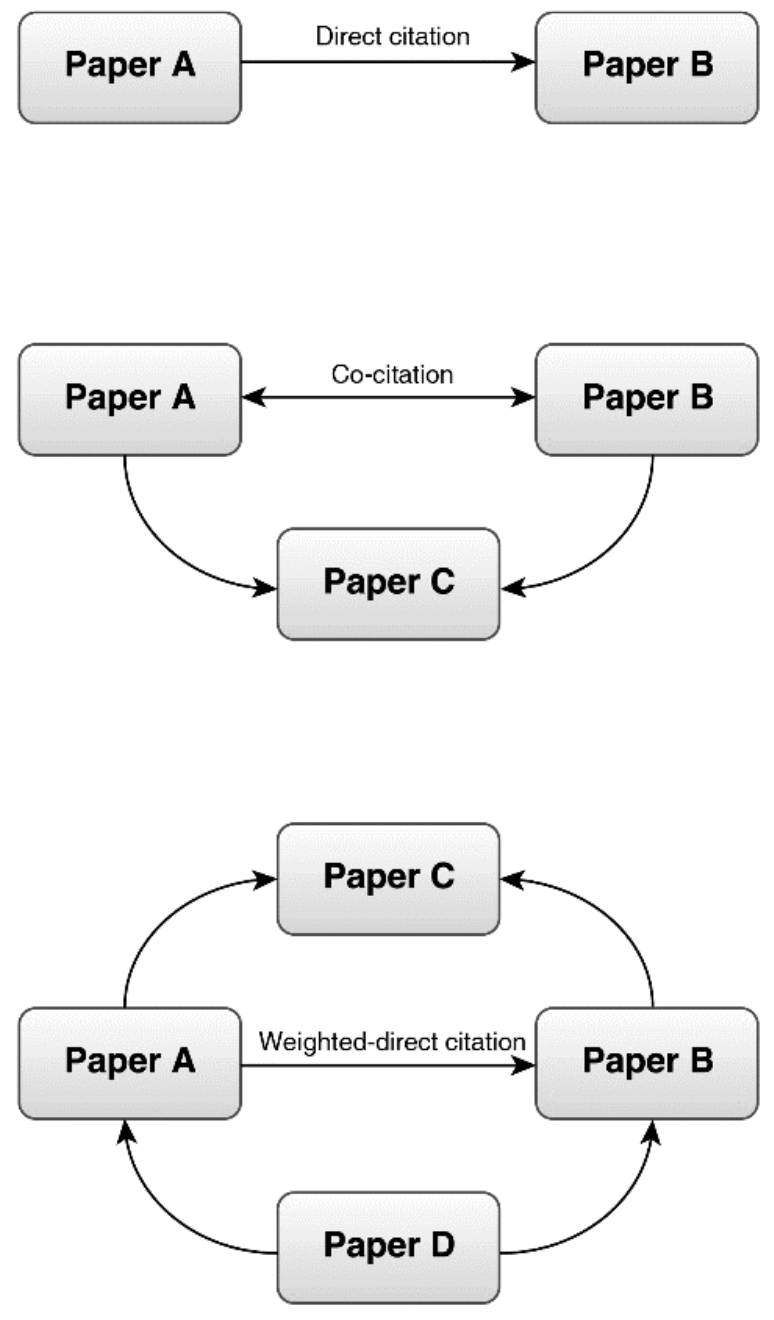

Figure 2. Illustration of direction citation, co-citation and weighted-direct citation analyses (Newell \& Cousins, 2015). 
Bibexcel was used to calculate the WDC value of the shortlisted WoS papers. Gephi was used for citation network visualisation and analysis. The modularity algorithm in Gephi offers an objective method to identify clusters, which groups nodes with more interactions into discrete clusters based on the citation links.

\subsection{Content analysis}

Each selected paper's content was examined in depth. Papers were grouped by type of analysis (whether it is a qualitative, quantitative, conceptual, or a review paper) and whether the paper conducted analysis by comparing their data spatially (mapping) or temporally (times-series/longitudinal). The unit of study (the smallest unit of analysis) was also classified based on the following categories:

1. Inter-region or city studies, which can be compared internationally and make use of aggregate data, such as city-wide vehicle distance travelled/fuel use to derive per capita indicators of fuel use;

2. Intra-urban studies, which usually use aggregated census data at small geographic levels within a city and which often create composite indicators for spatial analysis;

3. Individual level studies, which use disaggregated data that uses households, person or vehicles as the unit of study. These are often best at exploring the issue at its core, and allow calculation of more exact indicators that can measure actual transport affordability impacts caused by increasing fuel costs. However, data availability is often limited;

4. Urban-rural studies, which separate urban and rural samples and often make comparisons between the two; and

5. Aggregated studies, which use aggregate data in a single geographic unit (country, state, or city) without comparison of a similar kind. 
Other attributes were also extracted by reading each of the 320 papers. This included the mobility focus (car, public or active modes), the type of fuel price impacts, the preferred solution and the wider themes.

\section{Key findings and discussion}

\subsection{Overview of the historical and geographical trends}

Figure 3 compares the crude oil price with the number of shortlisted papers between 1972 and 2017. The volume of research publications largely follows the changes in fuel price. The 1974 oil crisis led to an initial spike in publications. During the oil glut (1985-2005), research interest waned and the number of papers fell. Publication rates rebounded after 2004 when oil prices began to rise again. Despite the sharp drop in oil prices in 2014 , papers covering this topic remain high, nearing 15-20 papers per year.

The geographical distribution of the shortlisted papers is illustrated in Table 2 and Figure 4. We split the dataset into the period before and after the year 2004, to highlight the recent high oil price period. There are signs that publications are shifting from traditional oil consumption countries (North America, Europe and Australasia) to newly industrialising ones, such as China and India. Along with increasing motorisaiton, increasing globalisation of academic research might play a role in this diffusion. Despite an increasing geographical spread, the volume of research remains disproportionately from Europe, North America and Australasia, which may limit transferability of findings to other regions. 


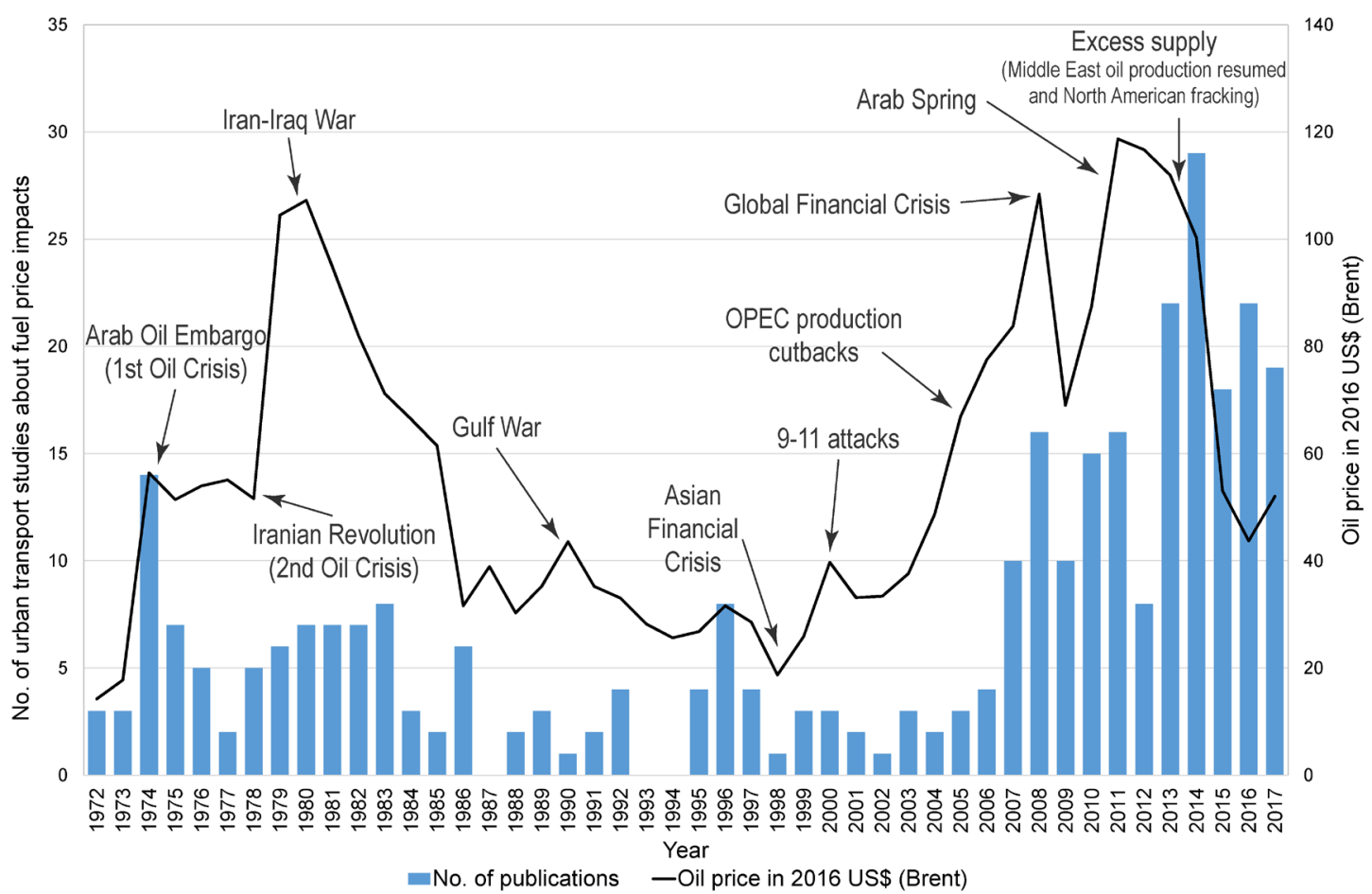

Figure 3. Comparison of the number of publications and crude oil price (in 2016 US\$) from 1972 to $2017(\mathrm{~N}=320)$.

Table 2. Worldwide distribution of the publications before and after $2004(\mathrm{~N}=320)$.

\begin{tabular}{|c|c|c|c|c|c|c|c|}
\hline \multirow[b]{2}{*}{ Region } & \multicolumn{2}{|c|}{ Before 2004} & \multicolumn{2}{|c|}{ After 2004} & \multirow{2}{*}{$\begin{array}{c}\text { Before } \\
\text { \& after } \\
2004 \\
\text { change } \\
(\%)\end{array}$} & \multicolumn{2}{|c|}{$\begin{array}{c}\text { All time } \\
(1970-2017)\end{array}$} \\
\hline & $\begin{array}{c}\text { F. } \\
\text { Count }\end{array}$ & $\begin{array}{c}\text { Share } \\
(\%)\end{array}$ & $\begin{array}{c}\text { F. } \\
\text { Count }\end{array}$ & $\begin{array}{c}\text { Share } \\
(\%)\end{array}$ & & $\begin{array}{c}\text { F. } \\
\text { Count }\end{array}$ & $\begin{array}{c}\text { Share } \\
(\%)\end{array}$ \\
\hline North America (USA + Canada) & 44.42 & 51.65 & 55.50 & 35.58 & 24.95 & 99.92 & 41.29 \\
\hline Europe & 19.92 & 23.16 & 44.50 & 28.53 & 123.43 & 64.42 & 26.62 \\
\hline Australasia (Australia + New Zealand) & 12.58 & 14.63 & 24.08 & 15.44 & 91.39 & 36.67 & 15.15 \\
\hline Asia & 6.92 & 8.04 & 24.00 & 15.38 & 246.99 & 30.92 & 12.78 \\
\hline Africa & 1.33 & 1.55 & 4.83 & 3.10 & 262.50 & 6.17 & 2.55 \\
\hline Central \& South Americas (incl. Mexico) & 0.83 & 0.97 & 3.08 & 1.98 & 270.00 & 3.92 & 1.62 \\
\hline All Geographical & 86 & 100.00 & 156 & 100.00 & 81.40 & 242 & 100.00 \\
\hline All Geographical & 86 & 68.25 & 156 & 80.41 & 81.40 & 242 & 75.63 \\
\hline Non-Geographical & 40 & 31.75 & 38 & 19.59 & -5.00 & 78 & 24.38 \\
\hline Total & 126 & 100.00 & 194 & 100.00 & 53.97 & 320 & 100.00 \\
\hline
\end{tabular}

F. Count refers to fractional count. Each study has a value of 1 , which can be divided if it is covering more than one continent. 


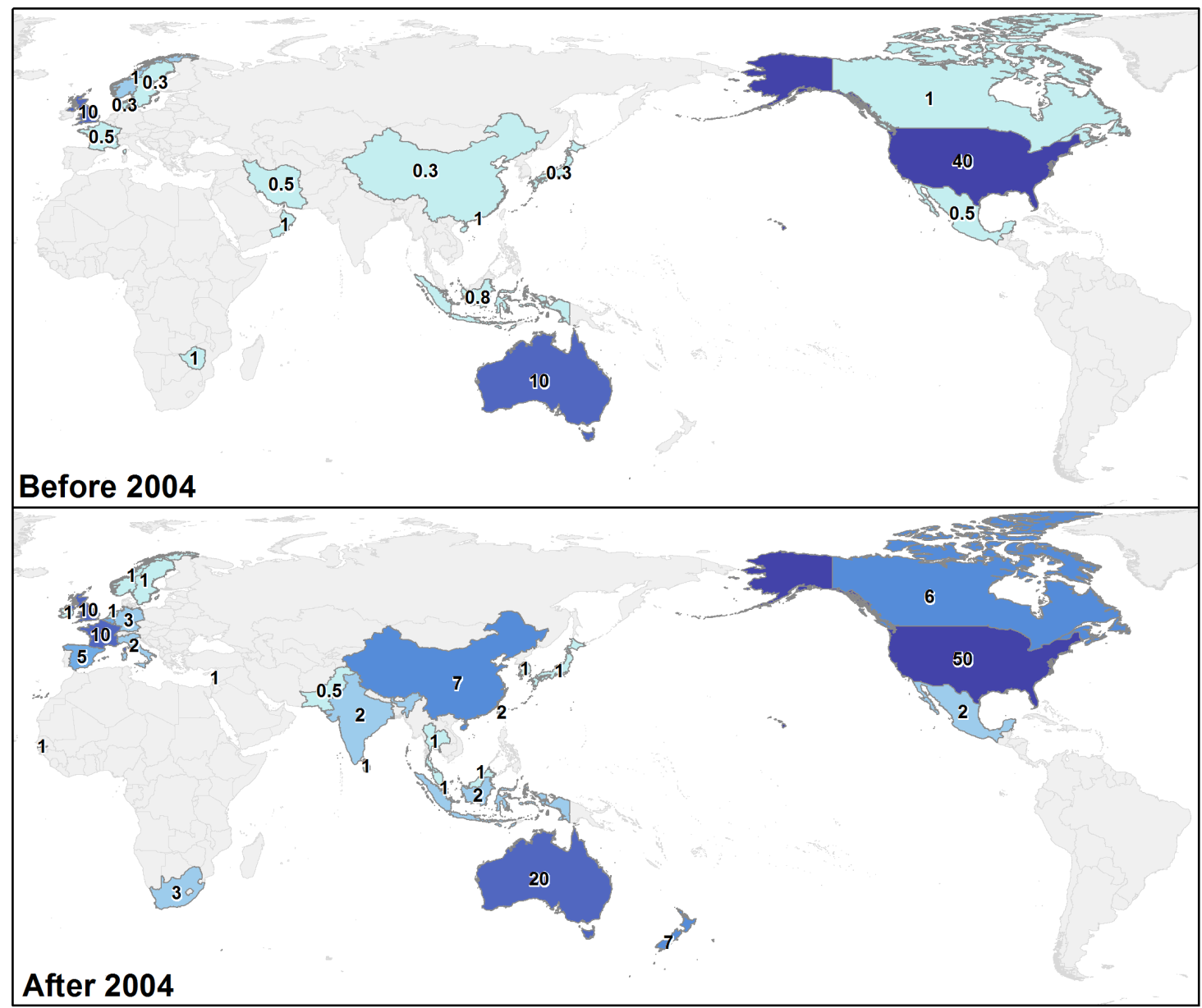

Publications on urban fuel price change impacts - by country/territory (fractional count)

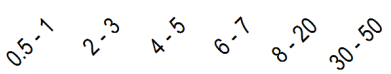

Figure 4. Worldwide country/territory distribution of the number of shortlisted publications with identifiable geographic coverage $(n=206)$, before and after 2004 .

\subsection{Citation network analysis and key research clusters}

Using the citation network analysis method outlined in Section 2, Figure 5 visualises the linkages between the shortlisted WoS papers. Larger nodes (circles) mean the publication has a higher WDC, a measure of citation influence. Thicker edges (arrows) means the paper is being co-cited more often and the direction denotes the direct citation flow, where the arrowhead is the paper being cited. The publication map is generated by the Proportional Yifan Hu algorithm offered in Gephi, which positions the nodes based on the relative intensity of citation relationship. Based on the network community detection algorithm in Gephi, eight major clusters were identified. Minor Clusters, with less than 
five papers are grouped as Cluster 0 for illustrative purposes. The naming of the clusters was determined by subsequent content analysis. Table 3 provides the summary statistics for these clusters. Here, those without any citations in the shortlist are also grouped as No Internal Cites. Tables 4, 5, 6, 7 and 8 summarise the percentages of the content analysis based on the paper's research methodology, key themes and geographical coverage.

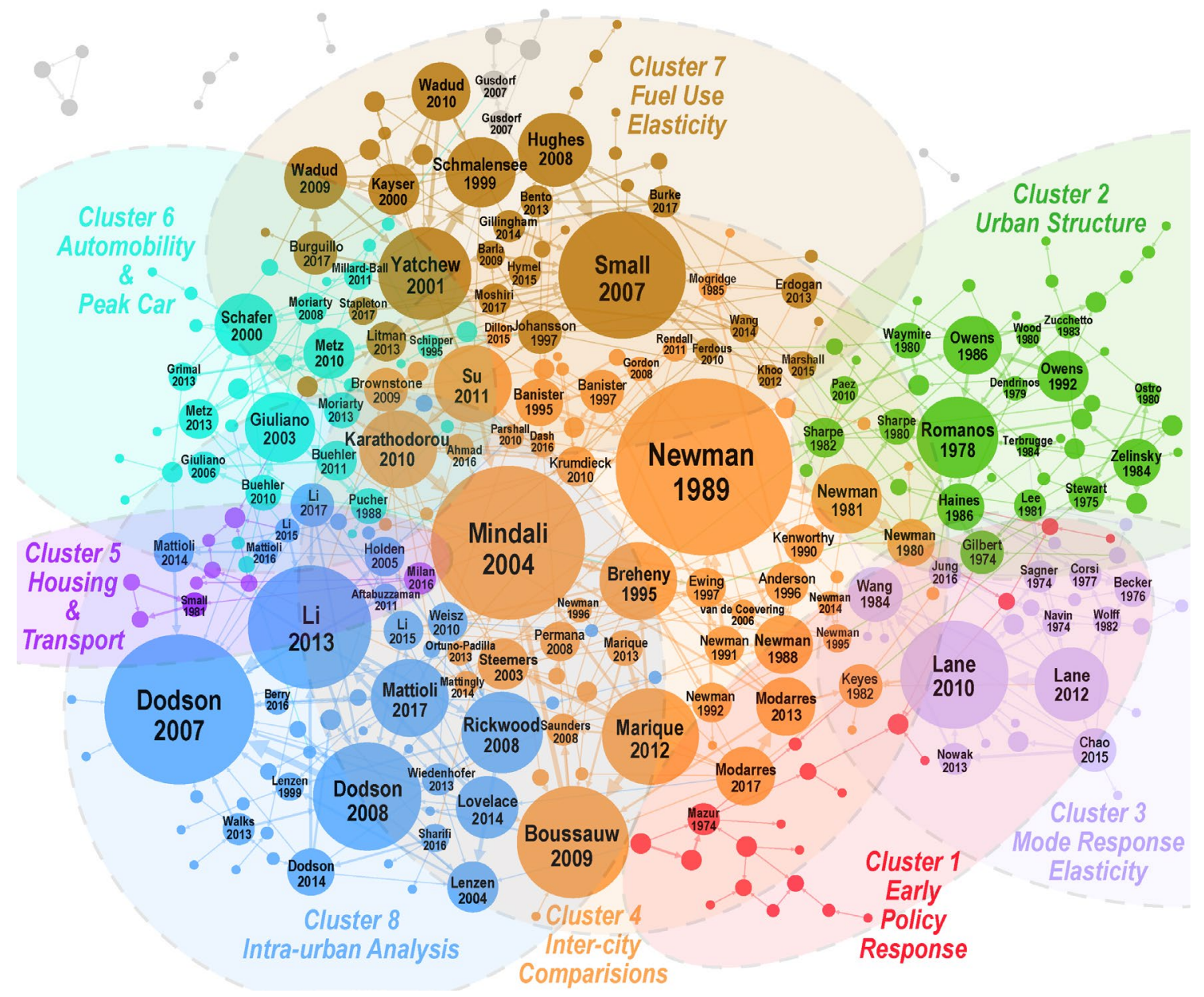

Figure 5. Bibliometric map showing the citation network $(\mathrm{N}=320)$.

A node (circle) refers to a publication. The size of a node is determined by the WDC score, which shows the relative citation influence of the publication. Only the nodes with a WDC score over 5 are labelled, showing the first author and year only. The edge (arrow) refers to the direct citation flow between two publications. The arrowhead indicates the source paper. Edge thickness reflects the number of co-cites between two publications. Clusters are coloured differently and are shaded by oblique dotted ovals of the widest extent of nodes. These ovals show a representative "sphere of influence" of a cluster. Minor Clusters (less than 5 nodes) are coloured in grey. 
Table 3. Summary of the clusters and their key information.

\begin{tabular}{clcccc}
\hline No. & \multicolumn{1}{c}{$\begin{array}{c}\text { Literature } \\
\text { clusters }\end{array}$} & $\begin{array}{c}\text { No. of } \\
\text { publications }\end{array}$ & Median year & Mode year & Std. dev. \\
\hline 1 & Early Policy Response & 20 & 1974 & 1974 & 7.86 \\
2 & Urban Structure & 41 & 1981 & $1978^{*}$ & 8.73 \\
3 & Mode Response Elasticity & 26 & 1985 & 1974 & 17.79 \\
4 & Inter-city Comparisons & 60 & 2008 & 2013 & 11.81 \\
5 & Housing and Transport & 9 & 2010 & $1979^{*}$ & 16.66 \\
6 & Automobility and Peak Car & 34 & 2010 & $2008^{*}$ & 9.91 \\
7 & Vehicle Fuel Elasticity & 39 & 2013 & 2017 & 5.15 \\
8 & Intra-urban Analysis & 47 & 2014 & 2016 & 6.05 \\
0 & Minor Clusters (Total) & 15 & 2009 & 2014 & 10.60 \\
- & No internal cites & 29 & 2012 & 2008 & 8.58 \\
\hline & Total & $\mathbf{3 2 0}$ & $\mathbf{2 0 0 8}$ & $\mathbf{2 0 1 4}$ & $\mathbf{1 5 . 0 6}$ \\
\hline
\end{tabular}

Citation count as of January 2018

* For clusters with multiple mode years, only the earliest year is shown.

Table 4. Summary of the methods used in the publications.

\begin{tabular}{|c|c|c|c|c|c|c|c|}
\hline No. & $\begin{array}{l}\text { Quantitative } \\
\text { modelling }\end{array}$ & $\begin{array}{l}\text { Quantitative } \\
\text { analysis }\end{array}$ & $\begin{array}{l}\text { Quan+Qual } \\
\text { (Mixed) }\end{array}$ & $\begin{array}{l}\text { Qualitative } \\
\text { analysis }\end{array}$ & $\begin{array}{l}\text { Literature } \\
\text { review }\end{array}$ & $\begin{array}{c}\text { Theoryl } \\
\text { policyl } \\
\text { conceptual }\end{array}$ & Total \\
\hline 1 & $\begin{array}{r}15.0 \% \\
(3)\end{array}$ & $\begin{array}{r}20.0 \% \\
(4)\end{array}$ & $\begin{array}{r}10.0 \% \\
(2)\end{array}$ & $\begin{array}{r}0.0 \% \\
(0)\end{array}$ & $\begin{array}{r}10.0 \% \\
(2)\end{array}$ & $\begin{array}{r}45.0 \% \\
(9)\end{array}$ & $\begin{array}{r}100 \% \\
(20)\end{array}$ \\
\hline 2 & $\begin{array}{r}26.8 \% \\
(11)\end{array}$ & $\begin{array}{r}12.2 \% \\
(5)\end{array}$ & $\begin{array}{r}7.3 \% \\
\text { (3) }\end{array}$ & $\begin{array}{r}4.9 \% \\
(2)\end{array}$ & $\begin{array}{r}2.4 \% \\
(1)\end{array}$ & $\begin{array}{r}46.3 \% \\
(19)\end{array}$ & $\begin{array}{r}100 \% \\
(41)\end{array}$ \\
\hline 3 & $\begin{array}{r}73.1 \% \\
(19)\end{array}$ & $\begin{array}{r}11.5 \% \\
(3)\end{array}$ & $\begin{array}{r}0.0 \% \\
(0)\end{array}$ & $\begin{array}{r}0.0 \% \\
(0)\end{array}$ & $\begin{array}{r}0.0 \% \\
(0)\end{array}$ & $\begin{array}{r}15.4 \% \\
(4)\end{array}$ & $\begin{array}{r}100 \% \\
(26)\end{array}$ \\
\hline 4 & $\begin{array}{r}43.3 \% \\
(26)\end{array}$ & $\begin{array}{r}31.7 \% \\
(19)\end{array}$ & $\begin{array}{r}5.0 \% \\
(3)\end{array}$ & $\begin{array}{r}0.0 \% \\
(0)\end{array}$ & $\begin{array}{r}3.3 \% \\
(2)\end{array}$ & $\begin{array}{r}16.7 \% \\
(10)\end{array}$ & $\begin{array}{r}100 \% \\
(60)\end{array}$ \\
\hline 5 & $22.2 \%$ & $33.3 \%$ & $\begin{array}{r}11.1 \% \\
(1)\end{array}$ & $0.0 \%$ & $0.0 \%$ & $33.3 \%$ & $\begin{array}{r}100 \% \\
\text { (9) }\end{array}$ \\
\hline 6 & $\begin{array}{r}32.4 \% \\
(11)\end{array}$ & $\begin{array}{r}20.6 \% \\
(7)\end{array}$ & $\begin{array}{r}11.8 \% \\
(4)\end{array}$ & $\begin{array}{r}2.9 \% \\
(1)\end{array}$ & $\begin{array}{r}2.9 \% \\
(1)\end{array}$ & $\begin{array}{r}29.4 \% \\
(10)\end{array}$ & $\begin{array}{r}100 \% \\
(34)\end{array}$ \\
\hline 7 & $\begin{array}{r}76.9 \% \\
(30)\end{array}$ & $\begin{array}{r}7.7 \% \\
(3)\end{array}$ & $\begin{array}{r}2.6 \% \\
\text { (1) }\end{array}$ & $\begin{array}{r}0.0 \% \\
(0)\end{array}$ & $\begin{array}{r}5.1 \% \\
(2)\end{array}$ & $\begin{array}{r}7.7 \% \\
(3)\end{array}$ & $\begin{array}{r}100 \% \\
(39)\end{array}$ \\
\hline 8 & $\begin{array}{r}29.8 \% \\
(14)\end{array}$ & $\begin{array}{r}38.3 \% \\
(18)\end{array}$ & $\begin{array}{r}0.0 \% \\
(0)\end{array}$ & $\begin{array}{r}4.3 \% \\
(2)\end{array}$ & $8.5 \%$ & $\begin{array}{r}19.1 \% \\
(9)\end{array}$ & $\begin{array}{r}100 \% \\
(47)\end{array}$ \\
\hline 0 & $\begin{array}{r}20.0 \% \\
(3)\end{array}$ & $\begin{array}{r}13.3 \% \\
(2)\end{array}$ & $\begin{array}{r}0.0 \% \\
(0)\end{array}$ & $\begin{array}{r}13.3 \% \\
(2)\end{array}$ & $\begin{array}{r}6.7 \% \\
(1)\end{array}$ & $\begin{array}{r}46.7 \% \\
\text { (7) }\end{array}$ & $\begin{array}{r}100 \% \\
(15)\end{array}$ \\
\hline - & $\begin{array}{r}48.3 \% \\
(14)\end{array}$ & $\begin{array}{r}6.9 \% \\
(2)\end{array}$ & $\begin{array}{r}3.4 \% \\
\text { (1) }\end{array}$ & $\begin{array}{r}6.9 \% \\
(2)\end{array}$ & $\begin{array}{r}6.9 \% \\
(2)\end{array}$ & $\begin{array}{r}27.6 \% \\
\text { (8) }\end{array}$ & $\begin{array}{r}100 \% \\
(29)\end{array}$ \\
\hline Total & $\begin{array}{r}41.6 \% \\
(133)\end{array}$ & $\begin{array}{r}20.6 \% \\
(66)\end{array}$ & $\begin{array}{r}4.7 \% \\
(15)\end{array}$ & $\begin{array}{r}2.8 \% \\
(9)\end{array}$ & $\begin{array}{r}4.7 \% \\
(15)\end{array}$ & $\begin{array}{r}25.6 \% \\
(82)\end{array}$ & $\begin{array}{r}100 \% \\
(320)\end{array}$ \\
\hline
\end{tabular}

Percentage values are compared with the total publications in the same cluster. Bracket refers to the number of papers. 
Table 5. Summary of the use of spatial or temporal analysis, and unit of study of the publications.

\begin{tabular}{|c|c|c|c|c|c|c|c|c|}
\hline \multirow[b]{2}{*}{ No. } & \multicolumn{4}{|c|}{ Method type } & \multicolumn{4}{|c|}{ Unit of study } \\
\hline & Mapping & Time-series & Inter-region & Inter-city & Intra-city & Individual & Urban-rural & Aggregated \\
\hline 1 & $5.0 \%$ & $15.0 \%$ & $5.0 \%$ & $0.0 \%$ & $5.0 \%$ & $20.0 \%$ & $0.0 \%$ & $20.0 \%$ \\
\hline 2 & $2.4 \%$ & $19.5 \%$ & $7.3 \%$ & $19.5 \%$ & $7.3 \%$ & $7.3 \%$ & $4.9 \%$ & $9.8 \%$ \\
\hline 3 & $0.0 \%$ & $65.4 \%$ & $3.8 \%$ & $26.9 \%$ & $7.7 \%$ & $11.5 \%$ & $3.8 \%$ & $38.5 \%$ \\
\hline 4 & $25.0 \%$ & $21.7 \%$ & $0.0 \%$ & $40.0 \%$ & $35.0 \%$ & $15.0 \%$ & $13.3 \%$ & $5.0 \%$ \\
\hline 5 & $66.7 \%$ & $22.2 \%$ & $0.0 \%$ & $11.1 \%$ & $44.4 \%$ & $11.1 \%$ & $22.2 \%$ & $11.1 \%$ \\
\hline 6 & $2.9 \%$ & $41.2 \%$ & $35.3 \%$ & $17.6 \%$ & $2.9 \%$ & $14.7 \%$ & $5.9 \%$ & $8.8 \%$ \\
\hline 7 & $15.4 \%$ & $69.2 \%$ & $20.5 \%$ & $5.1 \%$ & $23.1 \%$ & $48.7 \%$ & $30.8 \%$ & $5.1 \%$ \\
\hline 8 & $42.6 \%$ & $10.6 \%$ & $2.1 \%$ & $31.9 \%$ & $44.7 \%$ & $14.9 \%$ & $10.6 \%$ & $8.5 \%$ \\
\hline 0 & $13.3 \%$ & $6.7 \%$ & $0.0 \%$ & $20.0 \%$ & $6.7 \%$ & $13.3 \%$ & $20.0 \%$ & $6.7 \%$ \\
\hline- & $6.9 \%$ & $37.9 \%$ & $13.8 \%$ & $10.3 \%$ & $13.8 \%$ & $6.9 \%$ & $24.1 \%$ & $17.2 \%$ \\
\hline Total & $16.9 \%$ & $31.6 \%$ & $9.4 \%$ & $11.9 \%$ & $21.3 \%$ & $20.6 \%$ & $19.1 \%$ & $11.6 \%$ \\
\hline
\end{tabular}

More than one type can be considered in each publication, percentage values are compared with the total papers in the same cluster.

Table 6. Summary of the key themes by mode studied, type of fuel price impacts and proposed solutions.

\begin{tabular}{|c|c|c|c|c|c|c|c|c|c|c|}
\hline \multirow[b]{2}{*}{ No. } & \multicolumn{3}{|c|}{ Mode studied } & \multicolumn{4}{|c|}{ Type of fuel price effects } & \multicolumn{3}{|c|}{ Preferred solutions } \\
\hline & $\begin{array}{l}\text { Private } \\
\text { vehicles }\end{array}$ & $\begin{array}{l}\text { Public } \\
\text { transport }\end{array}$ & $\begin{array}{l}\text { Active } \\
\text { transport }\end{array}$ & Elasticity & $\begin{array}{l}\text { Cost of } \\
\text { travel }\end{array}$ & $\begin{array}{l}\text { Cost of } \\
\text { housing }\end{array}$ & $\begin{array}{c}\text { Cost of } \\
\text { domestic } \\
\text { energy } \\
\text { (e.g. } \\
\text { heating) }\end{array}$ & $\begin{array}{l}\text { Alternative } \\
\text { fuels }\end{array}$ & $\begin{array}{l}\text { Vehicle } \\
\text { fuel } \\
\text { efficiency }\end{array}$ & $\begin{array}{l}\text { Urban } \\
\text { form / } \\
\text { land } \\
\text { use mix }\end{array}$ \\
\hline 1 & $60.0 \%$ & $50.0 \%$ & $10.0 \%$ & $0.0 \%$ & $5.0 \%$ & $0.0 \%$ & $35.0 \%$ & $5.0 \%$ & $20.0 \%$ & $15.0 \%$ \\
\hline 2 & $36.6 \%$ & $34.1 \%$ & $4.9 \%$ & $12.2 \%$ & $22.0 \%$ & $7.3 \%$ & $7.3 \%$ & $9.8 \%$ & $9.8 \%$ & $80.5 \%$ \\
\hline 3 & $30.8 \%$ & $88.5 \%$ & $0.0 \%$ & $61.5 \%$ & $26.9 \%$ & $0.0 \%$ & $0.0 \%$ & $0.0 \%$ & $7.7 \%$ & $23.1 \%$ \\
\hline 4 & $70.0 \%$ & $66.7 \%$ & $41.7 \%$ & $16.7 \%$ & $13.3 \%$ & $5.0 \%$ & $11.7 \%$ & $0.0 \%$ & $18.3 \%$ & $88.3 \%$ \\
\hline 5 & $44.4 \%$ & $22.2 \%$ & $11.1 \%$ & $33.3 \%$ & $55.6 \%$ & $44.4 \%$ & $0.0 \%$ & $0.0 \%$ & $11.1 \%$ & $77.8 \%$ \\
\hline 6 & $70.6 \%$ & $76.5 \%$ & $38.2 \%$ & $14.7 \%$ & $20.6 \%$ & $2.9 \%$ & $0.0 \%$ & $14.7 \%$ & $38.2 \%$ & $70.6 \%$ \\
\hline 7 & $46.2 \%$ & $30.8 \%$ & $10.3 \%$ & $74.4 \%$ & $12.8 \%$ & $7.7 \%$ & $0.0 \%$ & $5.1 \%$ & $38.5 \%$ & $64.1 \%$ \\
\hline 8 & $48.9 \%$ & $44.7 \%$ & $19.1 \%$ & $6.4 \%$ & $40.4 \%$ & $23.4 \%$ & $25.5 \%$ & $6.4 \%$ & $19.1 \%$ & $63.8 \%$ \\
\hline 0 & $26.7 \%$ & $13.3 \%$ & $13.3 \%$ & $6.7 \%$ & $40.0 \%$ & $33.3 \%$ & $13.3 \%$ & $20.0 \%$ & $20.0 \%$ & $73.3 \%$ \\
\hline- & $24.1 \%$ & $34.5 \%$ & $10.3 \%$ & $24.1 \%$ & $37.9 \%$ & $6.9 \%$ & $3.4 \%$ & $27.6 \%$ & $20.7 \%$ & $41.4 \%$ \\
\hline Total & $49.1 \%$ & $50.0 \%$ & $19.1 \%$ & $24.7 \%$ & $24.4 \%$ & $10.0 \%$ & $10.0 \%$ & $8.1 \%$ & $21.3 \%$ & $63.8 \%$ \\
\hline
\end{tabular}

More than one type can be considered in each publication, percentage values are compared with the total papers in the same cluster.

Table 7. Summary of wider implications.

\begin{tabular}{|c|c|c|c|c|c|c|c|c|}
\hline \multirow[b]{2}{*}{ No. } & \multicolumn{8}{|c|}{ Wider implications of fuel price impacts } \\
\hline & $\begin{array}{l}\text { Disadvantage } \\
\text { / equity }\end{array}$ & Accessibility & $\begin{array}{l}\text { Health } \\
\text { / wellbeing }\end{array}$ & $\begin{array}{l}\text { Finance } \\
\text { / debt }\end{array}$ & Economy & $\begin{array}{l}\text { Carbon } \\
\text { emissions }\end{array}$ & Peak oil & Peak car \\
\hline 1 & $25.0 \%$ & $0.0 \%$ & $5.0 \%$ & $0.0 \%$ & $20.0 \%$ & $5.0 \%$ & $5.0 \%$ & $0.0 \%$ \\
\hline 2 & $4.9 \%$ & $7.3 \%$ & $2.4 \%$ & $0.0 \%$ & $7.3 \%$ & $2.4 \%$ & $7.3 \%$ & $0.0 \%$ \\
\hline 3 & $7.7 \%$ & $3.8 \%$ & $3.8 \%$ & $0.0 \%$ & $3.8 \%$ & $7.7 \%$ & $0.0 \%$ & $0.0 \%$ \\
\hline 4 & $10.0 \%$ & $20.0 \%$ & $3.3 \%$ & $0.0 \%$ & $0.0 \%$ & $20.0 \%$ & $11.7 \%$ & $0.0 \%$ \\
\hline 5 & $44.4 \%$ & $44.4 \%$ & $0.0 \%$ & $33.3 \%$ & $11.1 \%$ & $0.0 \%$ & $0.0 \%$ & $0.0 \%$ \\
\hline 6 & $14.7 \%$ & $14.7 \%$ & $11.8 \%$ & $0.0 \%$ & $2.9 \%$ & $38.2 \%$ & $11.8 \%$ & $17.6 \%$ \\
\hline 7 & $23.1 \%$ & $12.8 \%$ & $15.4 \%$ & $0.0 \%$ & $5.1 \%$ & $12.8 \%$ & $7.7 \%$ & $5.1 \%$ \\
\hline 8 & $42.6 \%$ & $14.9 \%$ & $6.4 \%$ & $10.6 \%$ & $10.6 \%$ & $25.5 \%$ & $29.8 \%$ & $0.0 \%$ \\
\hline 0 & $13.3 \%$ & $0.0 \%$ & $0.0 \%$ & $0.0 \%$ & $20.0 \%$ & $20.0 \%$ & $33.3 \%$ & $6.7 \%$ \\
\hline- & $13.8 \%$ & $10.3 \%$ & $6.9 \%$ & $3.4 \%$ & $24.1 \%$ & $24.1 \%$ & $0.0 \%$ & $0.0 \%$ \\
\hline Total & $18.4 \%$ & $12.5 \%$ & $6.3 \%$ & $2.8 \%$ & $8.4 \%$ & $17.5 \%$ & $11.6 \%$ & $2.8 \%$ \\
\hline
\end{tabular}

More than one type can be considered in each publication, percentage values are compared with the total papers in the same cluster. 
Table 8. Geographic coverage of papers by clusters.

\begin{tabular}{ccrrrrrr}
\hline No. & $\begin{array}{c}\text { North } \\
\text { America } \\
\text { (USA + } \\
\text { Canada) }\end{array}$ & Europe & $\begin{array}{c}\text { Australasia } \\
\text { (Australia + } \\
\text { New Zealand) }\end{array}$ & \multicolumn{1}{c}{ Asia } & Africa & $\begin{array}{c}\text { Central \& } \\
\text { South } \\
\text { Americas } \\
\text { (incl. Mexico) }\end{array}$ & $\begin{array}{c}\text { Non- } \\
\text { Geographical }\end{array}$ \\
\hline 1 & $45.8 \%$ & $0.8 \%$ & $5.8 \%$ & $5.8 \%$ & $0.8 \%$ & $0.8 \%$ & $40.0 \%$ \\
2 & $28.5 \%$ & $11.4 \%$ & $12.6 \%$ & $2.8 \%$ & $0.4 \%$ & $2.8 \%$ & $41.5 \%$ \\
3 & $73.1 \%$ & $3.8 \%$ & $0.0 \%$ & $11.5 \%$ & $0.0 \%$ & $0.0 \%$ & $11.5 \%$ \\
4 & $22.4 \%$ & $34.0 \%$ & $14.0 \%$ & $14.0 \%$ & $0.3 \%$ & $0.3 \%$ & $15.0 \%$ \\
5 & $11.1 \%$ & $11.1 \%$ & $55.6 \%$ & $0.0 \%$ & $0.0 \%$ & $0.0 \%$ & $22.2 \%$ \\
6 & $12.5 \%$ & $33.1 \%$ & $3.2 \%$ & $18.4 \%$ & $1.0 \%$ & $2.5 \%$ & $29.4 \%$ \\
7 & $67.3 \%$ & $10.9 \%$ & $0.6 \%$ & $8.3 \%$ & $0.0 \%$ & $0.0 \%$ & $12.8 \%$ \\
8 & $13.7 \%$ & $26.4 \%$ & $26.4 \%$ & $7.3 \%$ & $2.5 \%$ & $2.5 \%$ & $21.3 \%$ \\
0 & $15.6 \%$ & $22.2 \%$ & $0.0 \%$ & $2.2 \%$ & $13.3 \%$ & $0.0 \%$ & $46.7 \%$ \\
- & $22.1 \%$ & $20.4 \%$ & $10.9 \%$ & $13.5 \%$ & $7.5 \%$ & $1.4 \%$ & $24.1 \%$ \\
\hline Total & $31.2 \%$ & $20.1 \%$ & $11.5 \%$ & $9.7 \%$ & $1.9 \%$ & $1.2 \%$ & $24.4 \%$ \\
\hline
\end{tabular}

Based on fractional count - each publication has a value of 1 , which the value is divided when the publication is covering more than one continent(s).

\subsection{Content analysis based on cluster membership}

We found the clusters represent diverse studies encompassing various research methods and problem framing. In this subsection, we outline the eight major clusters identified: Cluster 1 (Policy Response) comprises mostly early publications dated in the 1970s. A majority are theory, policy or conceptual works, and suggest policy responses to the sudden oil price increase during the 1970s Oil Shocks (Mazur \& Rosa, 1974). This is followed by Cluster 2 (Urban Structure) where the links between energy, and transport and land use were first beginning to be recognised. Romanos' (1978) paper concerning urban form change for energy conservation is the most influential in this cluster (WDC $=$ 20). The locational mismatch of residence and workplace, resulting in longer travel distances and higher fuel consumption, was also recognised (G. Gilbert \& Dajani, 1974; Waymire \& Waymire, 1980). The choice of urban forms (centralised, decentralised or polycentric) in reducing travel distances was debated (Owens, 1986; Sharpe, 1982; Zucchetto, 1983).

Research then focused more on how travel behaviour responds to fuel price changes. Two major type of elasticity studies - Clusters 3 and 7 emerged. Both are characterised by the preference of time-series modelling studies that measure elasticity. Situated on the lower- 
right corner is Cluster 3 (Mode Response Elasticity) which contains earlier studies about the response to higher fuel prices by mode shifting. The key variables include public transport ridership (Lane, 2010) and mode share (Nizlek \& Duckstein, 1974). Most of the papers in Cluster 3 are aggregated at the city level due to the nature of public transport data. On the other hand, Cluster 7 (Vehicle Fuel Elasticity) has a greater focus on quantitative modelling studies using disaggregated individual level (i.e. personal/household or vehicle-based) data. The usual variables analysed are fuel consumption (Wadud, Graham, \& Noland, 2010), vehicle distance travelled (Small \& Van Dender, 2007; Wang \& Chen, 2014), car ownership (Johansson \& Schipper, 1997) or traffic flow (Burke, Batsuuri, \& Yudhistira, 2017). Many studies in Cluster 7 involved urban vs. rural issues and income elasticity analysis. However, the spatial resolution is usually wide, covering country, state or city levels with less emphasis on intra-urban comparison.

The focus then shifts to the urban level determinants of fuel use, as a more nuanced understanding of how urban form and land use promote car use (or dependence) emerged. This forms the conceptual basis for Cluster 4 (Inter-city Comparisons), the largest cluster with 60 publications. Building from the urban structure studies in Cluster 2, a series of global inter-city assessments using transport parameters and fuel use forms the bulk of this cluster. The seminal work of Newman \& Kenworthy (1989) has the highest WDC score (of 45). However, their findings were strongly contested (Mees, 2009; Mindali, Raveh, \& Salomon, 2004). Subsequent work continues this line of research with varied interpretations and improvements (Karathodorou et al., 2010). Cluster 4 also contains intra-urban analysis, as many papers divided their study area into inner and outer urban areas for inter-city comparison. With better data availability, some studies mapped an entire country, offering both inter- and intra-urban comparison (Boussauw, Neutens, \& 
Witlox, 2012). In general, these studies lend support to an urban and land use approach to deal with car dependence and price impacts. The promotion of non-motorised transport solutions is also the strongest in Cluster 4.

Cluster 5 (Housing and Transport) moves on from the urban comparison studies to look at the role of the trade-off between housing and transport cost (Milan \& Creutzig, 2016). The papers in this cluster mostly use mapping analysis and intra-city spatial comparison. Compared to the other clusters, Cluster 5 shows the greatest concern for the costs of travel and housing, disadvantage/equity, accessibility and issues of finance and debt.

Cluster 6 (Automobility and Peak Car) tends not to focus on cities, but on national or worldwide statistics of automobile use. Carbon emissions and vehicle fuel efficiency themes feature strongly in Cluster 6. Many studies here focus on the determinants of automobile ownership or usage, using national comparisons (Buehler, 2011; Giuliano \& Narayan, 2003). The notion of "peak car" (or more accurately, a peak in a community's vehicle distance travelled per capita) has been observed in a few highly developed western countries or cities (Bastian, Börjesson, \& Eliasson, 2016; Metz, 2010, 2013; Millard-Ball \& Schipper, 2011; Stokes, 2013), possibly due to economic recession (Klein \& Smart, 2017), oil prices (Stapleton, Sorrell, \& Schwanen, 2017; Wadud \& Baierl, 2017) or other factors. Car restraint policy and public transport investments may explain the slower motorisation rates now being seen in cities such as Beijing (Gao \& Newman, 2018). However, most developing countries are still motorising due to economic growth and a growing aspiration to drive (Pojani, 2016).

Finally, Cluster 8 (Intra-urban Analysis) contains a recent set of studies, mostly covering the recent period of higher oil prices (2005 to 2014). These focus on "peak oil", transport disadvantage, and equity issues. As with Cluster 5, mapping analysis featured strongly. The most influential paper in this cluster is Dodson and Sipe's (2007) development of a 
spatial composite indicator to reveal "oil vulnerability" in Australian cities. Another approach used was to estimate urban "energy requirements", which typically uses input and output tables to assess intra-urban differences in average domestic and transport fuel use (Wiedenhofer, Lenzen, \& Steinberger, 2013). Later research has looked at the sociospatial effects of fuel efficiency improvements in the private vehicle fleet ( $\mathrm{Li}$, Dodson, \& Sipe, 2015). Most recently these studies have turned to household level fuel expenditures as a measure of economic stress (Berry et al., 2016; Mattioli, Lucas, \& Marsden, 2017). In assessing these clusters, we found diverse research approaches and problem framing of the issue of fuel price effects on urban transport. We also observed some extent of geographical concentration within clusters (see Table 7). This implies research of similar types has a higher tendency to cite papers within the same continent. To summarise the clusters further, three distinctive research "traditions" can be identified. The first is the Elasticity tradition represented by Clusters 3 and 8, which tends to be based on quantitative modelling and uses time series and aggregated datasets at an individual level unit of study. These studies mostly look at North American cases. The second tradition is Car Dependence, represented by Cluster 4 and 6, which tends to use broad aggregated datasets at national, state or city level, and is less likely to use small area (census tract) or individual level data. This tradition focuses on the relationship of urban form and car dependence and is more likely to look at European cases. Finally, Clusters 6 and 9 represent the Spatial Equity tradition, which has a higher tendency to use mapping-based intra-city comparisons and places less emphasis on time-series changes. Cluster 6 is found to be most represented in Australasia, while Cluster 8 is most represented by both Europe and Australasian cases. Non-western countries are less represented in our datasets. This could be due to the focus of English language publications and our focus on the WoS database. Nevertheless, Asian studies are more likely to be in Cluster 6 or 4 . Whereas 
African studies are mostly located in Minor Clusters, looking at issues such as gender issues (Fofana, 2015) and rural energy access (Schiffer, 2016) which were not large enough to form major clusters via our methods.

\section{Implications and future research directions}

\subsection{Methodological considerations}

A key finding from this analysis is that the perspective and approach of studies into energy, mobility, and urban development has varied considerably over time, with an inconsistent focus on the problem to be addressed and the methods used in pursuing solutions to that problem. Elasticity studies identify balancing supply and demand for petroleum as the key problem and then focus strongly on quantitative modelling as the technique to discover how to best achieve that balance, offering an empirical basis for price-based policies, such as fuel taxes or incentives to spur fuel efficiency. Inter-city studies adopt a spatial perspective and thus recognise fuel price increase impacts as a symptom of inefficient land uses, and advocate for "compact cities" to make oil saving public/active transport more feasible. These studies appear to play a central role, as shown by their location at the heart of Figure 5, as they are being cited widely by most other clusters. These inter-city papers are currently acting as bridges for intellectual communication. Intra-urban studies focus on the socio-spatial inequity across urban space, recognising that areas that offer better non-car accessibility usually also feature higher housing costs. Conversely, cheaper housing is often available only in more car dependent and "oil vulnerable" locations.

The next generation of studies on fuel price transport impacts would benefit from integrating the energy problems under consideration and reconciling their methodological differences accordingly. These differences could be rooted in problem framing and 
methodological choices for the research. In future, inter-disciplinary approaches seem particularly promising. We note distinctive differences between the spatially-focused Equity (Clusters 5 and 8) and temporally-focused Elasticity approaches (Clusters 3 and 7), which our citation network mapping reveals are cleaved apart (Figure 5). Bringing these traditions together, encouraging researchers to co-learn from one-another, and connecting their insights, could all yield fruitful advances in understanding. Indeed, only a handful of studies appear that have considered both spatial and temporal approaches in the past (Gillingham, 2014; Motte-Baumvol, Massot, \& Byrd, 2009; Viguié, Hallegatte, \& Rozenberg, 2014; Walks, 2013). More of this is to be encouraged.

Another observation revealed in Table 3 is that most of the studies are either quantitative modelling or econometric analysis. Only a small number are purely qualitative or mixed studies. Though qualitative methods have their limitations, they are valuable in providing insights about how individuals and communities manage with the disadvantages they suffer from fuel price impacts. They can also reveal why individuals respond in the ways that they do.

Advances in technology also offer new 'solutions' to the problems collecting data on fuel use, transport and geo-spatial location. Shifts in travel data collection towards more passive approaches using mobile devices (Kaufman, 2017), and the potential to trigger surveys at key times on those same devices (such as when an individual has completed what appears to be a long motoring trip, or appears to have visited at petrol station) is under-explored in this sub-field of research. Such methods could produce cost-effective collection of key quantitative and qualitative data items.

\subsection{New trends that affect fuel price impact studies}

Apart from epistemological and methodological concerns, current fuel impact studies still largely measure automobile dependence through variables that capture the use of the 
automobile and its summary impacts. Researchers need to adjust to developments occurring in the transport field that may soon render previous fuel impact measures obsolete, or which may only capture a small portion of the total impact. In that sense, transport research can be seen as competing "regimes", in which the dominant three are: 1) car-based; 2) public-transport; and, 3) non-motorised, with niche innovations emerging rapidly (Moradi \& Vagnoni, 2018). For car-based research regimes, technological innovations in fuel efficiency (Vyas, Santini, \& Johnson, 2009) or electrification (Weiss, Dekker, Moro, Scholz, \& Patel, 2015) are being widely explored which flow through to policy proposals being provided to governments that narrowly focus on these concerns. Fuel impact studies should take account of improving fuel efficiency or hybrid/electric vehicle (EV) uptake. This has already been observed in more recent intra-urban studies (Li, Sipe, \& Dodson, 2013). Nevertheless, the "EV-ability" of households, including the ease of obtaining/purchasing an $\mathrm{EV}$, the ease of use and recharging in the city, and the availability of renewable energy (e.g., solar panels) has not so far been well assessed. The precipitous rise of electric bikes and especially the emergence of electric scooter share systems in 2017-18, show that there are other disruptions to urban mobility from electrification that require investigation in terms of possible fuel price impact (Hwang, 2010; Weinert, Ma, \& Cherry, 2007).

Further, the "disruptive" developments of autonomous vehicles (Ohnemus \& Perl, 2016) and ride-sharing (Cetin \& Deakin, 2017) adds significant uncertainty to the future demand for oil-fuelled mobility. There is a lack of consensus on whether these developments will lead to either reduced or increased car use (Bergman, Schwanen, \& Sovacool, 2017; Sheller \& Urry, 2016) with angst over how many kilometres taxi-like rideshare companies are now doing on the streets of many cities. There is also some scepticism that cars of whatever fuel source are not that all that useful in some cities, and especially in 
activity centres and downtown areas. Even autonomous vehicles may not help solve the spatial challenges of car dependence and may still cause negative externalities such as road safety or congestion.

Other 'solutions' to be explored include the use of ICT (information communication technology) for mobility substitution, including new forms of telecommuting, or virtualisation of work without leaving home, and the rise of suburban co-working hubs in many cities. It is uncertain whether these trends will replace or reduce travel, as recent studies have shown ICT may even further induce travel demand due to new opportunities (Cohen-Blankshtain \& Rotem-Mindali, 2016).

There is also a need for policy makers to receive better information on the true costs of automobile travel, with research needed to more fully flesh out these issues (Ferreira, Bertolini, \& Næss, 2017). Fuel dependency has almost never been considered in costbenefit appraisals of transport infrastructure investments. Nor have issues such as the health disbenefits (other than road trauma) of driving. Mapping of "oil vulnerability" has been highly influential in the field, as shown in Figure 5. But monetising these issues and incorporating them into conventional transport economics has proven difficult.

Further, with increasing uncertainty due to energy transition and technological changes, traditional "predict and plan" based on present-day needs must be replaced with more adaptive planning and infrastructure that can withstand the test of time (Givoni \& Perl, 2017).

\subsection{Equity concerns remain important}

Transport cost burdens are unevenly distributed across geographical space, a notion explored most obviously in Clusters 5 and 8 . But the issue of transport (in)equity is often overlooked in wider transport policy (Di Ciommo \& Shiftan, 2017). Price mechanisms that would raise the costs of automobile travel to a sufficiently high level (e.g., congestion 
charges, fuel taxes) might be effective, but they are likely to be inequitable in many cities due to current dependence on the car (Moriarty, 2016). Fuel price effects vary at different income levels (Wang \& Chen, 2014), as lower income households are likely to restrict mobility to necessary trips, whilst higher income counterparts can either afford discretionary travel, or invest in better fuel efficiency. While the land-use approach to reducing car dependence is often advocated by compact city theorists, housing-transport mismatch remains an issue for many cities, with the poor distributed to outer-suburban locations and prohibitive rents in locations with good non-car accessibility (Cao \& Hickman, 2017; Wells, 2012). The adoption of a robust public transport regime (e.g. in Singapore or Hong Kong) that mandates access to high-quality public transport in lowerincome housing estates has proven effective. But such policies might be less politically or socially acceptable in countries with a greater proportion of their population in rural or suburban areas and with greater democratic expression (Leung, Burke, Perl, \& Cui, 2018). Similarly, there are considerable differences in fuel tax/subsidies policy across nations. While fuel (or emissions) taxes present an effective way to restrain vehicle use or to promote fuel efficiency (Rodier, 2009), their future effectiveness will likely diminish due to the rise of electric vehicles and improving overall fuel-efficiency. Distance-based pricing of transport might help resolve the limitations of fuel tax, yet equity considerations remain important and taxing car use remains politically unpopular (Duncan, Nadella, Giroux, Bowers, \& Graham, 2017). Exploring alternative taxation instruments in terms of the socio-spatial effects on transport equity appears a pressing concern.

\section{Concluding remarks}

Our main contribution is in demonstrating the diverse, but inchoate body of research examining energy-related impacts on transport and land use, via the first systematic 
review of the field. Over two generations, progress has occurred in understanding the relationship between oil-based transport fuels, socio-economic impacts of mobility, and the spatial dynamics of urban development. It is hoped this assessment will provide a substantively informed basis for understanding what has been learned to date, and offer useful guidance about where and how future research on energy, transport and land use should proceed in order to generate more robust and holistic knowledge. This paper could serve as a first step toward linking the clusters and the research perspectives that have been identified. The limitations of the study include not being able to consider nonEnglish sources, which may omit other viewpoints and insights. Our bibliographic analysis only considered journal articles due to database limitations. The study focused on passenger transport as there are relatively few freight studies in this field (Cui, Dodson, \& Hall, 2015).

\section{References}

An, F., Gordon, D., He, H., Kodjak, D., \& Rutherford, D. (2007). Passenger Vehicle Greenhouse Gas and Fuel Economy Standards: A Global Update. The International Council on Clean Transportation.

Bastian, A., Börjesson, M., \& Eliasson, J. (2016). Explaining "peak car" with economic variables. Transportation Research Part A: Policy and Practice, 88, 236-250. https://doi.org/10.1016/j.tra.2016.04.005

Bergman, N., Schwanen, T., \& Sovacool, B. K. (2017). Imagined people, behaviour and future mobility: Insights from visions of electric vehicles and car clubs in the United Kingdom. Transport Policy, 59, 165-173. https://doi.org/10.1016/j.tranpol.2017.07.016

Berry, A., Jouffe, Y., Coulombel, N., \& Guivarch, C. (2016). Investigating fuel poverty in the transport sector: Toward a composite indicator of vulnerability. Energy Research \& Social Science. https://doi.org/10.1016/j.erss.2016.02.001

Bonilla, D. (2009). Fuel demand on UK roads and dieselisation of fuel economy. Energy Policy, 37(10), 3769-3778. https://doi.org/10.1016/j.enpol.2009.07.016

Boussauw, K., Neutens, T., \& Witlox, F. (2012). Relationship between Spatial Proximity and Travel-to-Work Distance: The Effect of the Compact City. Regional Studies, 46(6), 687-706. https://doi.org/10.1080/00343404.2010.522986 
Buehler, R. (2011). Determinants of transport mode choice: a comparison of Germany and the USA. Journal of Transport Geography, 19(4), 644-657. https://doi.org/10.1016/j.jtrangeo.2010.07.005

Burke, P. J., Batsuuri, T., \& Yudhistira, M. H. (2017). Easing the traffic: The effects of Indonesia's fuel subsidy reforms on toll-road travel. Transportation Research Part A: Policy and Practice, 105, 167-180. https://doi.org/10.1016/j.tra.2017.08.003

Cao, M., \& Hickman, R. (2017). Car dependence and housing affordability: An emerging social deprivation issue in London? Urban Studies. https://doi.org/10.1177/0042098017712682

Cetin, T., \& Deakin, E. (2017). Regulation of taxis and the rise of ridesharing. Transport Policy. https://doi.org/10.1016/j.tranpol.2017.09.002

Cohen-Blankshtain, G., \& Rotem-Mindali, O. (2016). Key research themes on ICT and sustainable urban mobility. International Journal of Sustainable Transportation, 10(1), 9-17. https://doi.org/10.1080/15568318.2013.820994

Colgan, J. D. (2014). Oil, Domestic Politics, and International Conflict. Energy Research \& Social Science, 1, 198-205. https://doi.org/10.1016/j.erss.2014.03.005

Coventry, D. H. (2011). Peak oil and significant change for rural Australia. Rural Society, 20(3), 235-243. https://doi.org/10.5172/rsj.20.3.235

Cui, J., Dodson, J., \& Hall, P. V. (2015). Planning for Urban Freight Transport: An Overview. Transport Reviews, 35(5), 583-598. https://doi.org/10.1080/01441647.2015.1038666

Currie, G., \& Delbosc, A. (2011). Transport Disadvantage: A Review. In G. Currie (Ed.), New Perspectives and Methods in Transport and Social Exclusion Research (pp. 15-25). Emerald Group Publishing Limited. https://doi.org/10.1108/9781780522012

Czako, J. A. (2015, February). Mobility Pricing in Road Transport Paradigm Shift to improve congestion, environment, road safety, and financing. Presented at the 2nd Annual ASECAP Marketing Workshop, Paris, France.

Denmark, D. (1998). The Outsiders: Planning and Transport Disadvantage. Journal of Planning Education and Research, 17(3), 231-245. https://doi.org/10.1177/0739456X9801700304

Di Ciommo, F., \& Shiftan, Y. (2017). Transport equity analysis. Transport Reviews, 37(2), 139-151. https://doi.org/10.1080/01441647.2017.1278647

Dodson, J., Burke, M., Sipe, N., \& Perl, A. (2016). The social meaning of “access". In E. Sclar, M. Lönnroth, \& C. Wolmar (Eds.), Improving urban access: new approaches to funding transport investment (pp. 40-60). London; New York, NY: Routledge Taylor \& Francis Group.

Dodson, J., \& Sipe, N. (2007). Oil vulnerability in the Australian city: assessing socioeconomic risks from higher urban fuel prices. Urban Studies, 44(1), 37-62. https://doi.org/10.1080/00420980601023810

Duncan, D., Nadella, V., Giroux, S., Bowers, A., \& Graham, J. D. (2017). The road mileage user-fee: Level, intensity, and predictors of public support. Transport Policy, 53, 70-78. https://doi.org/10.1016/j.tranpol.2016.09.002

Ewing, R., Hamidi, S., Tian, G., Proffitt, D., Tonin, S., \& Fregolent, L. (2018). Testing Newman and Kenworthy's Theory of Density and Automobile Dependence. Journal of Planning Education and Research, 38(2), 167-182. https://doi.org/10.1177/0739456X16688767 
Ferreira, A., Bertolini, L., \& Næss, P. (2017). Immotility as resilience? A key consideration for transport policy and research. Applied Mobilities, 2(1), 16-31. https://doi.org/10.1080/23800127.2017.1283121

Figueroa, M., Lah, O., Fulton, L. M., McKinnon, A., \& Tiwari, G. (2014). Energy for Transport. Annual Review of Environment and Resources, 39(1), 295-325. https://doi.org/10.1146/annurev-environ-031913-100450

Fofana, I. (2015). Gender Analysis of the Policy Responses to High Oil Prices: A Case Study of South Africa. Feminist Economics, 21(3), 216-240. https://doi.org/10.1080/13545701.2015.1023330

Fuller, B., \& Romer, P. (2014). Urbanization as Opportunity. Washington, D.C., USA: The World Bank Sustainable Development Network.

Gao, Y., \& Newman, P. (2018). Beijing's Peak Car Transition: Hope for Emerging Cities in the $1.5^{\circ} \mathrm{C}$ Agenda. Urban Planning, 3(2), 82. https://doi.org/10.17645/up.v3i2.1246

Gilbert, G., \& Dajani, J. S. (1974). Energy, urban form and transportation policy. Transportation Research, 8(4-5), 267-276. https://doi.org/10.1016/00411647(74)90046-X

Gilbert, R., \& Perl, A. (2012). Transport Revolutions: Moving People and Freight Without Oil (2nd ed.). London: Earthscan.

Gillingham, K. (2014). Identifying the elasticity of driving: Evidence from a gasoline price shock in California. Regional Science and Urban Economics, 47, 13-24. https://doi.org/10.1016/j.regsciurbeco.2013.08.004

Giuliano, G., \& Narayan, D. (2003). Another Look at Travel Patterns and Urban Form: The US and Great Britain. Urban Studies, 40(11), 2295-2312. https://doi.org/10.1080/0042098032000123303

Givoni, M., \& Perl, A. (2017). Rethinking Transport Infrastructure Planning to Extend Its Value over Time. Journal of Planning Education and Research, 0739456X1774119. https://doi.org/10.1177/0739456X17741196

Goodwin, P., Dargay, J., \& Hanly, M. (2004). Elasticities of Road Traffic and Fuel Consumption with Respect to Price and Income: A Review. Transport Reviews, 24(3), 275-292. https://doi.org/10.1080/0144164042000181725

Hamilton, J. D. (2013). History of Oil Shocks. In R. E. Parker \& R. Whaples (Eds.), Routledge handbook of major events in economic history (pp. 239-266). London; New York: Routledge.

Hickman, R., \& Banister, D. (2014). Transport, climate change and the city. London; New York: Routledge, Taylor \& Francis Group.

Hine, J., \& Mitchell, F. (2001). Better for Everyone? Travel Experiences and Transport Exclusion. Urban Studies, 38(2), 319-332. https://doi.org/10.1080/00420980020018619

Hwang, J. J. (2010). Sustainable transport strategy for promoting zero-emission electric scooters in Taiwan. Renewable and Sustainable Energy Reviews, 14(5), 13901399. https://doi.org/10.1016/j.rser.2010.01.014

Jeekel, H. (2014). Social exclusion, vulnerable groups and driving forces: Towards a social research based policy on car mobility. Case Studies on Transport Policy, 2(2), 96-106. https://doi.org/10.1016/j.cstp.2014.06.005

Johansson, O., \& Schipper, L. (1997). Measuring the Long-Run Fuel Demand of Cars: Separate Estimations of Vehicle Stock, Mean Fuel Intensity, and Mean Annual Driving Distance. Journal of Transport Economics and Policy, 31(3), 277-292. 
Karathodorou, N., Graham, D. J., \& Noland, R. B. (2010). Estimating the effect of urban density on fuel demand. Energy Economics, 32(1), 86-92. https://doi.org/10.1016/j.eneco.2009.05.005

Kaufman, B. (2017). Exploration of New Methods in Long Distance Transportation Data Collection and Tourism Travel in Vermont (Vol. Graduate College Dissertations and Theses. 809). Burlington, VT, USA: University of Vermont. Retrieved from https://scholarworks.uvm.edu/graddis/809/

Kenworthy, J. R., Laube, F. B., Newman, P., \& Barter, P. A. (1999). An international sourcebook of automobile dependence in cities 1960-1990. Boulder: University Press of Colorado.

Kerschner, C., Prell, C., Feng, K., \& Hubacek, K. (2013). Economic vulnerability to Peak Oil. Global Environmental Change, 23(6), 1424-1433. https://doi.org/10.1016/j.gloenvcha.2013.08.015

Khan, M. I. (2017). Falling oil prices: Causes, consequences and policy implications. Journal of Petroleum Science and Engineering, 149, 409-427. https://doi.org/10.1016/j.petrol.2016.10.048

Khayesi, M., \& Amekudzi, A. A. (2011). Kingdon's multiple streams model and automobile dependence reversal path: the case of Curitiba, Brazil. Journal of Transport Geography, 19(6), 1547-1552. https://doi.org/10.1016/j.jtrangeo.2011.06.012

Klein, N. J., \& Smart, M. J. (2017). Millennials and car ownership: Less money, fewer cars. Transport Policy, 53, 20-29. https://doi.org/10.1016/j.tranpol.2016.08.010

Lane, B. W. (2010). The relationship between recent gasoline price fluctuations and transit ridership in major US cities. Journal of Transport Geography, 18(2), 214-225. https://doi.org/10.1016/j.jtrangeo.2009.04.002

Leung, A., Burke, M., Perl, A., \& Cui, J. (2018). The peak oil and oil vulnerability discourse in urban transport policy: A comparative discourse analysis of Hong Kong and Brisbane. Transport Policy, 65, 5-18. https://doi.org/10.1016/j.tranpol.2017.03.023

Li, T., Dodson, J., \& Sipe, N. (2015). Exploring Social and Spatial Patterns in Private Vehicle Fuel Efficiency: a case study of Brisbane and Sydney, Australia. Australian Geographer, 46(2), 217-233. https://doi.org/10.1080/00049182.2015.1020994

Li, T., Sipe, N., \& Dodson, J. (2013). Investigating Private Motorised Travel and Vehicle Fleet Efficiency: Using New Data and Methods to Reveal Socio-Spatial Patterns in Brisbane, Australia. Geographical Research, 51(3), 269-278. https://doi.org/10.1111/1745-5871.12001

Litman, T. (2018). Evaluating Transportation Equity Guidance For Incorporating Distributional Impacts in Transportation Planning. Victoria, BC, Canada: Victoria Transport Policy Institute.

Lucas, K., Mattioli, G., Verlinghieri, E., \& Guzman, A. (2016). Transport poverty and its adverse social consequences. In Proceedings of the Institution of Civil Engineers - Transport. Thomas Telford (ICE Publishing).

Lyons, G., \& Chatterjee, K. (Eds.). (2002). Transport lessons from the fuel tax protests of 2000. Hampshire, Eng.; Burlington, VT: Ashgate.

Martens, K. (2012). Justice in transport as justice in accessibility: applying Walzer's 'Spheres of Justice' to the transport sector. Transportation, 39(6), 1035-1053. https://doi.org/10.1007/s11116-012-9388-7

Mattioli, G., Anable, J., \& Vrotsou, K. (2016). Car dependent practices: Findings from a sequence pattern mining study of UK time use data. Transportation Research 
Part A: Policy and Practice, 89, 56-72.

https://doi.org/10.1016/j.tra.2016.04.010

Mattioli, G., Lucas, K., \& Marsden, G. (2017). Transport poverty and fuel poverty in the UK: From analogy to comparison. Transport Policy, 59, 93-105. https://doi.org/10.1016/j.tranpol.2017.07.007

Mattioli, G., Wadud, Z., \& Lucas, K. (2018). Vulnerability to fuel price increases in the UK: A household level analysis. Transportation Research Part A: Policy and Practice, 113, 227-242. https://doi.org/10.1016/j.tra.2018.04.002

Mazur, A., \& Rosa, E. (1974). Energy and Life-Style. Science, 186(4164), 607-610. https://doi.org/10.1126/science.186.4164.607

Mees, P. (2009). Density delusion? Urban form and sustainable transport in Australian, Canadian and US cities. World Transport Policy and Practice, 15(2), 29-42.

Metz, D. (2010). Saturation of Demand for Daily Travel. Transport Reviews, 30(5), 659-674. https://doi.org/10.1080/01441640903556361

Metz, D. (2013). Peak Car and Beyond: The Fourth Era of Travel. Transport Reviews, 33(3), 255-270. https://doi.org/10.1080/01441647.2013.800615

Milan, B. F., \& Creutzig, F. (2016). Municipal policies accelerated urban sprawl and public debts in Spain. Land Use Policy, 54, 103-115. https://doi.org/10.1016/j.landusepol.2016.01.009

Millard-Ball, A., \& Schipper, L. (2011). Are We Reaching Peak Travel? Trends in Passenger Transport in Eight Industrialized Countries. Transport Reviews, 31(3), 357-378. https://doi.org/10.1080/01441647.2010.518291

Mindali, O., Raveh, A., \& Salomon, I. (2004). Urban density and energy consumption: A new look at old statistics. Transportation Research Part A: Policy and Practice, 38, 143-162. https://doi.org/10.1016/j.tra.2003.10.004

Moradi, A., \& Vagnoni, E. (2018). A multi-level perspective analysis of urban mobility system dynamics: What are the future transition pathways? Technological Forecasting and Social Change, 126, 231-243. https://doi.org/10.1016/j.techfore.2017.09.002

Moriarty, P. (2016). Reducing levels of urban passenger travel. International Journal of Sustainable Transportation, 10(8), 712-719. https://doi.org/10.1080/15568318.2015.1136364

Motte-Baumvol, B., Massot, M.-H., \& Byrd, A. M. (2009). Escaping Car Dependence in the Outer Suburbs of Paris. Urban Studies, 47(3), 604-619. https://doi.org/10.1177/0042098009349773

Newell, J. P., \& Cousins, J. J. (2015). The boundaries of urban metabolism: Towards a political-industrial ecology. Progress in Human Geography, 39(6), 702-728. https://doi.org/10.1177/0309132514558442

Newman, P., \& Kenworthy, J. R. (1989). Cities and automobile dependence: a sourcebook. Aldershot, Hants, UK; Brookfield, VT, US: Gower Technical.

Nizlek, M. C., \& Duckstein, L. (1974). A system model for predicting the effect of energy resources on urban modal split. Transportation Research, 8(4-5), 329334. https://doi.org/10.1016/0041-1647(74)90051-3

Ohnemus, M., \& Perl, A. (2016). Shared Autonomous Vehicles: Catalyst of New Mobility for the Last Mile? Built Environment, 42(4), 589-602. https://doi.org/10.2148/benv.42.4.589

Owens, S. (1986). Strategic planning and energy conservation. Town Planning Review, 57(1), 69. https://doi.org/10.3828/tpr.57.1.w374181612n1813t

Persson, O. (2010). Identifying research themes with weighted direct citation links. Journal of Informetrics, 4(3), 415-422. https://doi.org/10.1016/j.joi.2010.03.006 
Pojani, D. (2016). The urban transport crisis in emerging economies. New York, NY: Springer Science+Business Media.

Rendall, S., Page, S., Reitsma, F., Van Houten, E., \& Krumdieck, S. (2011).

Quantifying Transport Energy Resilience: Active Mode Accessibility.

Transportation Research Record: Journal of the Transportation Research

Board, 2242(1), 72-80. https://doi.org/10.3141/2242-09

Rodier, C. (2009). Review of International Modeling Literature: Transit, Land Use, and Auto Pricing Strategies to Reduce Vehicle Miles Traveled and Greenhouse Gas Emissions. Transportation Research Record: Journal of the Transportation Research Board, 2132, 1-12. https://doi.org/10.3141/2132-01

Romanos, M. C. (1978). Energy-price effects on metropolitan spatial structure and form. Environment and Planning A, 10(1), 93-104. https://doi.org/10.1068/a100093

Schiffer, A. (2016). Empowered, excited, or disenfranchised? Unveiling issues of energy access inequality and resource dependency in The Gambia. Energy Research \& Social Science, 18, 50-61. https://doi.org/10.1016/j.erss.2016.04.011

Schipper, L. (2011). Automobile use, fuel economy and CO2 emissions in industrialized countries: Encouraging trends through 2008? Transport Policy, 18(2), 358-372. https://doi.org/10.1016/j.tranpol.2010.10.011

Sharpe, R. (1982). Energy efficiency and equity of various urban land use patterns. Urban Ecology, 7(1), 1-18. https://doi.org/10.1016/0304-4009(82)90002-X

Sheller, M., \& Urry, J. (2016). Mobilizing the new mobilities paradigm. Applied Mobilities, 1(1), 10-25. https://doi.org/10.1080/23800127.2016.1151216

Small, K. A., \& Van Dender, K. (2007). Fuel Efficiency and Motor Vehicle Travel: The Declining Rebound Effect. The Energy Journal, 28(1), 25-51.

Social Exclusion Unit (SEU). (2003). Making the Connections: Final Report on Transport and Social Exclusion, London. London, UK: Cabinet Office, UK. Retrieved from http://www.socialexclusionunit.gov.uk/publications/reports/ntml/transportfinal/s ummary

Sperling, D., \& Gordon, D. (2009). Two billion cars: driving toward sustainability. Oxford; New York: Oxford University Press. Retrieved from http://public.eblib.com/choice/publicfullrecord.aspx? $\mathrm{p}=416014$

Stapleton, L., Sorrell, S., \& Schwanen, T. (2017). Peak car and increasing rebound: A closer look at car travel trends in Great Britain. Transportation Research Part D: Transport and Environment, 53, 217-233. https://doi.org/10.1016/j.trd.2017.03.025

Stokes, G. (2013). The Prospects for Future Levels of Car Access and Use. Transport Reviews, 33(3), 360-375. https://doi.org/10.1080/01441647.2013.800614

U.S. Energy Information Administration. (2016). International Energy Outlook 2016 With Projections to 2040. Washington DC, USA: U.S. Energy Information Administration (EIA), U.S. Department of Energy.

Van de Graaf, T., \& Verbruggen, A. (2015). The oil endgame: Strategies of oil exporters in a carbon-constrained world. Environmental Science \& Policy, 54, 456-462. https://doi.org/10.1016/j.envsci.2015.08.004

Viguié, V., Hallegatte, S., \& Rozenberg, J. (2014). Downscaling long term socioeconomic scenarios at city scale: A case study on Paris. Technological Forecasting and Social Change, 87, 305-324. https://doi.org/10.1016/j.techfore.2013.12.028 
Vyas, A. D., Santini, D. J., \& Johnson, L. R. (2009). Plug-In Hybrid Electric Vehicles ' Potential for Petroleum Use Reduction: Issues Involved in Developing Reliable Estimates.

Wadud, Z., \& Baierl, M. (2017). Explaining "peak car" with economic variables: A comment. Transportation Research Part A: Policy and Practice, 95, 381-385. https://doi.org/10.1016/j.tra.2016.11.002

Wadud, Z., Graham, D. J., \& Noland, R. B. (2010). Gasoline Demand with Heterogeneity in Household Responses. The Energy Journal, 31(1), 47-74.

Walks, A. (2013). Mapping the Urban Debtscape: The Geography of Household Debt in Canadian Cities. Urban Geography, 34(2), 153-187. https://doi.org/10.1080/02723638.2013.778647

Wang, T., \& Chen, C. (2014). Impact of fuel price on vehicle miles traveled (VMT): do the poor respond in the same way as the rich? Transportation, 41(1), 91-105. https://doi.org/10.1007/s11116-013-9478-1

Waymire, B., \& Waymire, E. (1980). Effects of rising energy prices on urban space. Regional Science and Urban Economics, 10(3), 407-422. https://doi.org/10.1016/0166-0462(80)90040-X

Weinert, J., Ma, C., \& Cherry, C. (2007). The transition to electric bikes in China: history and key reasons for rapid growth. Transportation, 34(3), 301-318. https://doi.org/10.1007/s11116-007-9118-8

Weiss, M., Dekker, P., Moro, A., Scholz, H., \& Patel, M. K. (2015). On the electrification of road transportation - A review of the environmental, economic, and social performance of electric two-wheelers. Transportation Research Part D: Transport and Environment, 41, 348-366. https://doi.org/10.1016/j.trd.2015.09.007

Wells, P. (2012). Converging transport policy, industrial policy and environmental policy: The implications for localities and social equity. Local Economy, 27(7), 749-763. https://doi.org/10.1177/0269094212455018

Wells, P., \& Xenias, D. (2015). From 'freedom of the open road' to 'cocooning': Understanding resistance to change in personal private automobility.

Environmental Innovation and Societal Transitions, 16, 106-119. https://doi.org/10.1016/j.eist.2015.02.001

Wiedenhofer, D., Lenzen, M., \& Steinberger, J. K. (2013). Energy requirements of consumption: Urban form, climatic and socio-economic factors, rebounds and their policy implications. Energy Policy, 63, 696-707. https://doi.org/10.1016/j.enpol.2013.07.035

Zucchetto, J. (1983). Energy and the future of human settlement patterns: Theory, models and empirical considerations. Ecological Modelling, 20(2-3), 85-111. https://doi.org/10.1016/0304-3800(83)90001-7 